\title{
ОПРЕДЕЛЕНИЕ ВЕРТИКАЛЬНОГО ГРАДИЕНТА СИЛЫ ТЯЖЕСТИ НА ТЕРРИТОРИЮ ЗАПАДНОЙ СИБИРИ
}

\section{Вадим Федорович Канушин}

Сибирский государственный университет геосистем и технологий, 630108, Россия, г. Новосибирск, ул. Плахотного, 10, кандидат технических наук, доцент кафедры космической и физической геодезии, тел. (913)983-77-26, e-mail: kanushinvf43.work.ssg@gmail.com

\section{Ирина Геннадьевна Ганагина}

Сибирский государственный университет геосистем и технологий, 630108, Россия, г. Новосибирск, ул. Плахотного, 10, кандидат технических наук, заведующая кафедрой космической и физической геодезии, тел. (913)956-46-42, e-mail: gam0209@yandex.ru

\section{Денис Николаевич Голдобин}

Сибирский государственный университет геосистем и технологий, 630108, Россия, г. Новосибирск, ул. Плахотного, 10, кандидат технических наук, старший преподаватель кафедры космической и физической геодезии, тел. (913)455-92-66, e-mail: phis.geo.sgga@gmail.com

Вертикальный градиент силы тяжести имеет большое практическое значение, его использование позволяет значительно расширить возможности по локализации и интерпретации гравитационных аномалий. Благодаря применению измеренного вертикального градиента силы тяжести на земной поверхности возможно повысить точность приведения к отсчетной поверхности измеренных значений ускорения силы тяжести, а также найти среднюю кривизну уровенной поверхности.

Для региональных исследований гравитационного поля предложено использовать определение вертикальной составляющей градиента силы тяжести с помощью разложения в ряд Фурье по системе сферических функций.

Приведены результаты вычисления вертикального градиента силы тяжести на территории Западной Сибири по гармоническим коэффициентам современной глобальной модели геопотенциала EIGEN-6C4. Приведены результаты сравнения вертикальных градиентов поля силы тяжести, вычисленными по гармоническим коэффициентам современных глобальных моделей геопотенциала, и с вертикальными градиентами, полученными из измерений высокоточными гравиметрами. Установлено, что отличия нормального значения от вычисленных вертикальных градиентов достигают 662,4 Э.

Ключевые слова: вертикальный градиент, глобальные модели геопотенциала, сферические функции, сила тяжести, гравиметрия

\section{DETERMINATION OF THE VERTICAL GRADIENT OF GRAVITY ON THE TERRITORY OF WESTERN SIBERIA}

\section{Vadim F. Kanushin}

Siberian State University of Geosystems and Technologies, 10, Plakhotnogo St., Novosibirsk, 630108, Russia, Ph. D., Associate Professor, Department of Space and Physical Geodesy, phone: (913)983-77-26, e-mail: kanushinvf43.work.ssg@gmail.com

\section{Irina G. Ganagina}

Siberian State University of Geosystems and Technologies, 10, Plakhotnogo St., Novosibirsk, 630108, Russia, Ph. D., Head of the Department of Space and Physical Geodesy, phone: (913)956-46-42, e-mail: gam0209@yandex.ru 


\section{Denis N. Goldobin}

Siberian State University of Geosystems and Technologies, 10, Plakhotnogo St., Novosibirsk, 630108, Russia, Ph. D., Senior Lecturer, Department of Space and Physical Geodesy, phone: (913)455-92-66, e-mail: phis.geo.sgga@gmail.com

The vertical gradient of gravity is of great practical importance, its use can significantly expand the possibilities for the localization and interpretation of gravitational anomalies. The use of the measured vertical gradient of gravity on the earth's surface will improve the accuracy of the measured values of the acceleration of gravity to the reference surface, as well as find the average curvature of the level surface.

For regional studies of the gravitational field, it is proposed to use the definition of the vertical component of the gravity gradient using the expansion in a Fourier series in a system of spherical functions.

The results of calculating the vertical gradient of gravity in the territory of Western Siberia from the harmonic coefficients of the modern global model of the EIGEN-6C4 geopotential are presented. The results of a comparison of the vertical gradients of the gravity field, calculated from the harmonic coefficients of modern global geopotential models, with the vertical gradients obtained from measurements by high-precision gravimeters are presented. It was found that the differences between the normal value and the calculated vertical gradients reach 662.4 Oe.

Keywords: vertical gradient, global geopotential models, spherical functions, gravity, gravimetry

\section{Введение}

В различные годы в отечественной и зарубежной литературе активно обсуждается вопрос о способах определения и применения вертикального градиента силы тяжести для решения задач наук о Земле $[2,3,6,7,9-11,13,14]$

$$
W_{z z}=\frac{\partial g}{\partial z}
$$

где $g$ - сила тяжести; $\mathrm{z}$ - ось топоцентрической прямоугольной системы координат (xyz) направлена по отвесной линии в надир.

Вертикальный градиент силы тяжести (ВГСТ) описывает изменение силы тяжести с высотой и имеет большое практическое значение. Его использование позволяет значительно расширить возможности по локализации и интерпретации гравитационных аномалий. По сравнению с аномалиями силы тяжести (АСТ) ВГСТ обладает большей разрешающей способностью, благодаря чему он широко применяется для выделения локальных аномалий в гравитационном поле Земли.

Применение измеренного ВГСТ на земной поверхности позволит повысить точность приведения к отсчетной поверхности измеренных значений ускорения силы тяжести (УСТ), а также найти среднюю кривизну уровенной поверхности. Особое значение приобретает знание вертикального градиента при измерении силы тяжести на подвижном основании (аэрогравиметрия, инерциальная 
гравиметрия и т.д.). С появлением в последние десятилетия высокоточных гравиметров основным методом измерения вертикального градиента является измерение силы тяжести на разной высоте. Расчеты, показывают, что такие измерения можно выполнять с погрешностью от 3 до 10 Э (Этвеш) при разности высот установки прибора порядка 3 м [4, 8, 12].

При интерпретации АСТ относительно редко используют измеренные значения вертикального градиента силы тяжести, а при редуцировании УСТ на отсчетную поверхность применяют нормальный вертикальный градиент, вычисляемый по следующей формуле [12, 13]

$$
\frac{\partial \gamma}{\partial h}=-\left(0.3087691-0.0004398 \sin ^{2} B\right)
$$

При малых значениях высот вторым членом формулы (2) можно пренебречь [12] и вычислять нормальный вертикальный градиент, принимая $\mathrm{B}=45^{\circ}$, как

$$
\frac{\partial \gamma}{\partial h}=-0.3086 \text { мГал/м. }
$$

Отклонения измеренного ВГСТ от его нормального значения на различных станциях наблюдений и даже площадях достигают значительных величин.

В таблице 1 приведены статистические параметры разностей между измеренными на 16 гравиметрических пунктах, расположенных на территории России, [1] и нормальными значениями ВГСТ, полученными по формуле

$$
\Delta W_{z z}=W_{z z}-U_{z z}
$$

где $U_{z z}=0,3086$ мГал - нормальный вертикальный градиент силы тяжести.

Из табл. 1 видно, что измеренные в различных пунктах значения вертикального градиента $W_{z z}$ значительно отличаются относительно нормального градиента $U_{z z}=3086$ Э.

Таблийа 1

Статистические параметры распределения разности $\Delta W_{z z}$

\begin{tabular}{|l|c|}
\hline \multicolumn{1}{|c|}{ Параметр } & Значение (Этвеш) \\
\hline Минимум & -686 \\
\hline Максимум & 1124 \\
\hline Диапазон & 1810 \\
\hline Среднее & 46 \\
\hline Стандартное отклонение & 360 \\
\hline Асимметрия & 1,043 \\
\hline Эксцесс & 3,246 \\
\hline
\end{tabular}


Отличия измеренных значений вертикального градиента от нормального обусловлены притяжением близ поверхностных возмущающих масс с аномальной плотностью, отличной от средней для земной коры, а также крупных инженерных сооружений в окрестности станции наблюдения. По величине вертикальный градиент силы тяжести превосходит остальные вторые производные потенциала силы тяжести, поэтому для его определения с погрешностью в 1 Э необходимо измерять ускорение силы тяжести с ошибкой $1 \cdot 10^{-9} \mathrm{M} / \mathrm{c}^{2} .[4,5,12]$. С увеличением высоты разность между измеренным и нормальным вертикальным градиентом убывает. Это связано с ослаблением влияния короткопериодических топографических эффектов и близ поверхностных аномальных масс.

В данной работе предложено для региональных исследований гравитационного поля использовать определение вертикальной составляющей градиента силы тяжести с помощью разложения в ряд Фурье по системе сферических функций. Приведены результаты вычисления вертикального градиента силы тяжести на территории Западной Сибири по гармоническим коэффициентам современной глобальной модели геопотенциала EIGEN-6C4.

\section{Эксперименты}

Используя данные глобальных моделей геопотенциала, можно вычислить вертикальный градиент с помощью разложения в ряд по сферическим функциям

$$
\begin{gathered}
W_{z z}=\frac{\partial^{2} W}{\partial r^{2}} \\
W_{z z}=\frac{f M}{r^{3}}\left[\sum_{n=0}^{N}(n+1)(n+2)\left(\frac{a_{e}}{r}\right)^{n} \sum_{m=0}^{n}\left(\bar{C}_{n m} \cos m \lambda+\bar{S}_{n m} \sin m \lambda\right) \bar{P}_{n m}(\sin \phi)\right]- \\
-\omega^{2} \cos ^{2} \phi
\end{gathered}
$$

где $\bar{C}_{n m}$ и $\bar{S}_{n m}$ - нормированные гармонические коэффициенты геопотенциала;

$\bar{P}_{n m}(\sin \phi)$ - нормированные присоединенные функции Лежандра степени $\mathrm{n}$ и порядка $\mathrm{m}$;

$f M$ - геоцентрическая гравитационная постоянная;

$a_{e}-$ большая полуось эллипсоида;

$r$ - геоцентрическое расстояние;

$\varphi$ - геоцентрическая широта;

$\lambda-$ географическая долгота.

По формуле (5) выполнено вычисление ВГСТ на территории Западной Сибири по данным глобальной модели геопотенциала EIGEN-6C4 с учетом сферических гармоник до степени $\mathrm{N}=2190$. Результаты вычисления ВГСТ на исследуемой территории приведены на рисунке. 


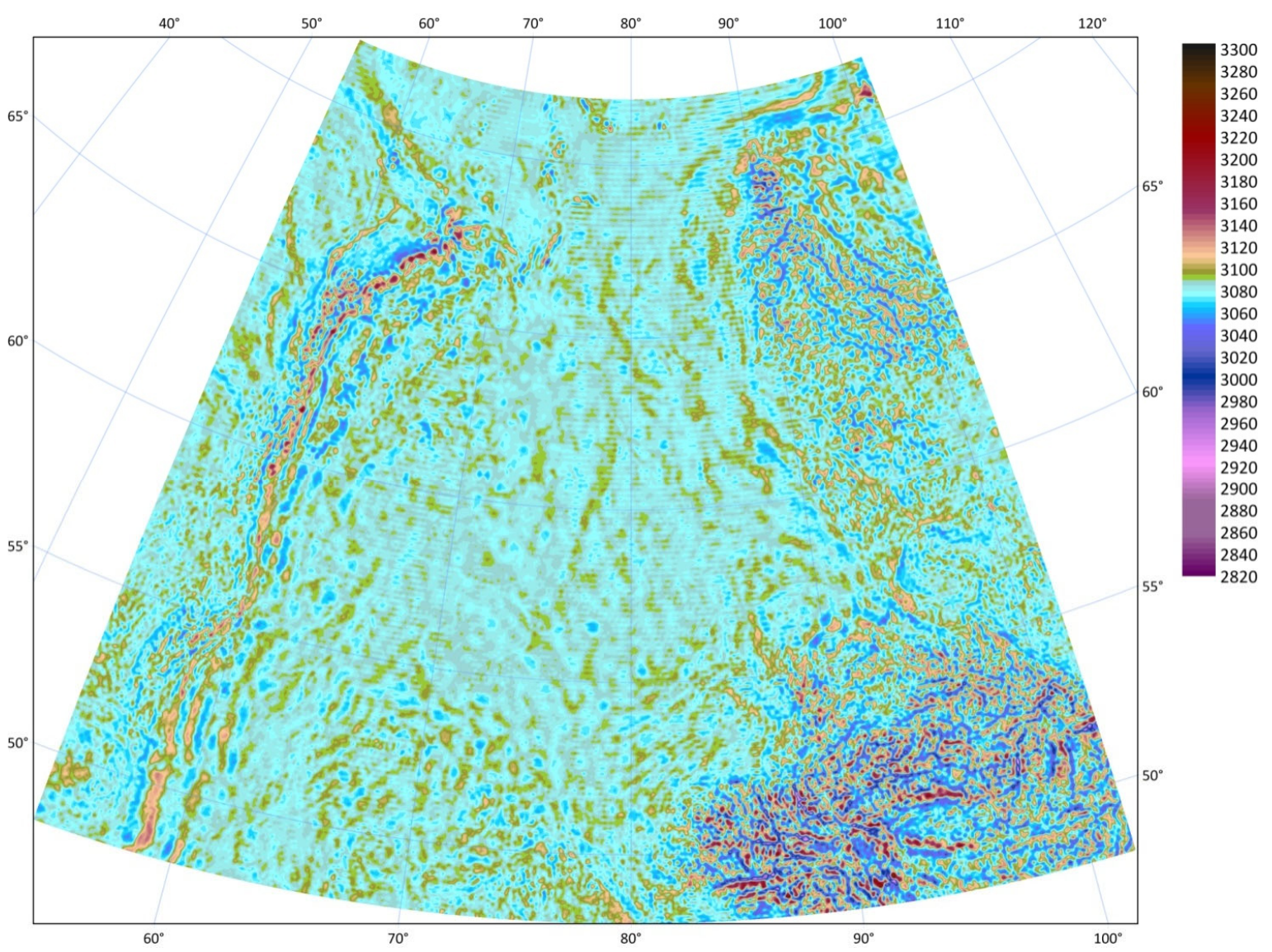

Картосхема вычисленных ВГСТ на территории Западной Сибири (в Этвешах)

В табл. 2 приведены статистические параметры распределения результатов сравнения ВГСТ, вычисленные по формуле (5), с полученными из измерений высокоточными гравиметрами на 16 гравиметрических пунктах, расположенных на исследуемой территории.

Таблииа 2

Статистические параметры распределения результатов сравнения ВГСТ, измеренными высокоточными гравиметрами и вычисленными по формуле (5)

\begin{tabular}{|l|c|}
\hline \multicolumn{1}{|c|}{ Параметр } & Значение (Этвеш) \\
\hline \multicolumn{1}{|c|}{1} & 2 \\
\hline Минимум & $-688,7$ \\
\hline Максимум & 217,5 \\
\hline Диапазон & 906,3 \\
\hline Среднее & $-24,5$ \\
\hline Стандартное отклонение & 260 \\
\hline Асимметрия & $-1,421$ \\
\hline Эксцесс & 1,200 \\
\hline
\end{tabular}


Анализируя распределение результатов сравнения ВГСТ, вычисленных по формуле (5), с полученными из измерений высокоточными гравиметрами на 16 гравиметрических пунктах, расположенных на исследуемой территории, можно сделать вывод о возможности использования при обработке гравиметрических измерений значения ВГСТ, вычисленные по современным глобальным моделям геопотенциала.

\section{Выводы. Обсуждения}

Полученные результаты исследования вертикального градиента силы тяжести в различных пунктах на изучаемой территории с привлечением данных глобальной модели геопотенциала EIGEN-6C4 позволяют сделать следующие выводы.

1. Измеренные в различных пунктах на исследуемой территории значения вертикального градиента силы тяжести значительно отличаются между собой и от нормального градиента (3086 Э).

2. Расхождения между вычисленными значениями ВГСТ, полученными по данным глобальной модели геопоенциала EIGEN-6C4, и измеренными высокоточными гравиметрами на пунктах наблюдений, достигают 688 Э, при среднеквадратическом отклонении 260 Э.

Поскольку реальные вертикальные градиенты силы тяжести на пунктах наблюдений значительно отличаются от нормального, что приводит к некорректной интерпретации гравитационных аномалий при его использовании, то при высокоточных измерениях силы тяжести и ее редуцировании предлагается определять вертикальный градиент силы тяжести с помощью разложения в ряд по сферическим функциям, используя гармонические коэффициенты современных ультравысокостепенных моделей геопотенциала.

\section{БИБЛИОГРАФИЧЕСКИЙ СПИСОК}

1. Кузьмин В.И., Голушко А.В. Результаты измерения градиентов ускорения силы тяжести на пунктах государственной гравиметрической сети. Вестник Сибирской государственной геодезической академии/СГГА.-Вып.1.-Новосибирск, 1996. - 146с.

2. Бычков С.Г., 2005, Особенности обработки результатов современной гравиметрической съемки: Геофизический вестник, 12, 9-13.

3. Антонов Ю.В., Силкин К.Ю., Черников К.С. Карта вертикального градиента силы тяжести центральной части Воронежского массива // Геофизика. - 2006. - № 3. - стр. 53 - 56.

4. Миронов В. С. Курс гравиразведки. Л.: Недра, 1972.- 512 с.

5. Маловичко А.К., Костицын В.И. Гравиразведка. - М.: Недра, 1992. - 357.

6. Ager C.A., Liard S.O. Vertical Gravity Gradient Surveys: Field Results and Interpretation in British Columbia, Canada // Geophysics. 1982. - V. 47. -№ 7. P. 919-91.

7. Faikicwisz Z. Gravity Vertical Measurements for the Detection of Small and Antropogenic form // Geophysics. 1976. - V. 41. - № 5. - P. 1016 - 1030.

8. Балавадзе Б.К. К методике определения вертикального градиента силы тяжести // Изв. АН СССР. Сер. геофиз. 1968. - Вып. 27. - стр. 54 - 56.

9. Thyissen-Bornemisza S., Stalklew W. Observation of the Vertical Gradient of Gravity in the Field // Geophysics. 1956. - V.21. - N3. - P. $771-741$. 
10. Антонов Ю.В., Силкин К.Ю., Черников К.С. Карта вертикального градиента силы тяжести центральной части Воронежского массива // Геофизика. - 2006. - № 3. - С. 53 - 56.

11. Ляндрес А.Ю. ООО «Гео Дейта консалтинг». Интерпретация аномалий гравитационного поля с применением значений его вертикального градиента. http://www.georazrez.ru 2008 стр.1-6.

12. Торге В. Гравиметрия: Пер. с англ.- М., Мир, 1999 - 429 с.

13. Бычков С.Г., Долгаль А.С., Симанов А.А. Вычисление аномалий силы тяжести при высокоточных гравиметрических съемках. Пермь, УрОРАН, 2015 - 142 с.

14. Kopaev A., Shteyman M., Vitushkin L. (1993) Repeated Measurements of Vertical Gravity Gradient. In: Montag H., Reigber C. (eds) Geodesy and Physics of the Earth. International Association of Geodesy Symposia, vol 112. Springer, Berlin, Heidelberg.

(C) В. Ф. Канушин, И. Г. Ганагина, Д. Н. Голдобин, 2021 